\title{
A hyperacute neurology team - transforming emergency neurological care
}

\author{
Authors: Arani Nitkunan, ${ }^{A}$ Bridget K MacDonald, ${ }^{A}$ Ajay Boodhoo, ${ }^{B}$ Andrew Tomkins, ${ }^{C}$ Caitlin Smyth, ${ }^{B}$ \\ Medina Southam ${ }^{B}$ and Fred Schon ${ }^{A}$
}

\begin{abstract}
We present the results of an 18-month study of a new model of how to care for emergency neurological admissions. We have established a hyperacute neurology team at a single district general hospital. Key features are a senior acute neurology nurse coordinator, an exclusively consultant-delivered service, acute epilepsy nurses, an acute neurophysiology service supported by neuroradiology and acute physicians and based within the acute medical admissions unit. Key improvements are a major increase in the number of patients seen, the speed with which they are seen and the percentage seen on acute medical unit before going to the general wards. We have shown a reduced length of stay and readmission rates for patients with epilepsy. Epilepsy accounted for $30 \%$ of all referrals. The cost implications of running this service are modest. We feel that this model is worthy of widespread consideration.
\end{abstract}

KEYWORDS: Emergency care, hyperacute, neurology, neurology specialist nurse

\section{Introduction}

There is increasing evidence that rapid diagnosis and treatment improve patient outcomes. This has perhaps most clearly been shown in the management of acute myocardial infarction and stroke, which has resulted in ambulances taking patients directly to specialist centres and hyperacute stroke units (HASUs) in the case of suspected stroke.

The optimal way to manage other acute neurological emergencies is not yet established even though they account for $19 \%$ of all non-stroke acute medical admissions. ${ }^{1}$ In 2011, the Association of British Neurologists proposed a 24-hour target for patients to be seen by neurologists after referral. ${ }^{2}$

One model is to set up hyperacute neurology units (HANUs) analogous to the stroke model; pilot studies were carried out in two London hospitals for a very limited period of 6 weeks in $2015 .^{3}$ The three proposed advantages of these units were:

Authors: ${ }^{A}$ consultant neurologist, Croydon University Hospital, Croydon, UK; ${ }^{B}$ specialist nurse, Croydon University Hospital, Croydon, UK; Clead clinical neurophysiologist, Croydon University Hospital, Croydon, UK
1 understanding the problem better, leading to quick, correct diagnosis

2 facilitating patient flow with quick referral to right team

3 achieving better outcomes - right decisions early with care plan.

We present an analogous study of an alternative model based at a single district general hospital (population 363,000), using similar targets - a hyperacute neurology team (HANT) running a 5-day service. This has been running now for about 22 months and we present 18 months of data unless stated otherwise. The HANT comprises six key features:

1 based within the acute medical admission unit (AMU) and the rapid access medical unit (RAMU)

2 coordinated by a senior acute neurology specialist nurse

3 exclusively consultant delivered

4 includes acute community epilepsy nurses

5 involves telephone and 'hot' clinic slots to facilitate early discharge

6 has an acute neurophysiology service attached.

Before establishing the current model, there was inconsistent cover for neurological referrals during consultant leave periods. A recent survey describes how varied the service is within the UK with $20 \%$ of acute hospitals having access to a neurologist for 3 or fewer days a week. ${ }^{4}$

\section{Methods}

\section{Members of the HANT}

\section{The HANT is composed of:}

$>$ four consultant neurologists (total 3.2 whole-time equivalent (WTE) sessions) - prior to November 2015, there were three consultants (2.6 WTE)

$>$ one full-time band 8 senior acute neurology unit coordinator

$>$ two band 7 full-time epilepsy nurses who see all epilepsy patients admitted to the acute service

$>$ an acute neurophysiology service provided by two part-time consultants (1 WTE) and four clinical physiologists (2.8 WTE)

$>$ one acute neuroimaging radiographer (magnetic resonance imaging/computerised tomography)

> a lead AMU consultant. 


\section{The consultant role in the HANT}

The four consultants each have a designated on-call day, including prospective cover for annual leave. Friday cover is on a 1:4 rota to cater for attendance at continuing medical education. If there are an excessive number of referrals, one of the other consultants will try to assist the on-call consultant.

\section{The role of the nurse coordinator}

Monday to Friday, the nurse coordinator attends the AMU at 9 and 11 am and takes all available neurological referrals. Patients can also be referred from the RAMU and ambulatory emergency care unit, which provides an accident and emergency service to GPs. Between 9 am and 12 pm the nurse coordinator sees these patients and records basic demographics, summarises clinical details (from the electronic notes) and records key investigation results, such as bloods tests, neuroimaging, electroencephalogram (EEG)/electromyogram (EMG) and lumbar puncture. They also carry out a preliminary clinical assessment.

From 12-5 pm, the nurse takes the consultant of the day to see each patient. This mainly occurs between 12 and $2.30 \mathrm{pm}$, but when required will be until $5 \mathrm{pm}$.

After the consultant has seen the patients, the nurse carries out the following:

$>$ documentation in electronic notes

$>$ ordering tests (if essential)

$>$ arranging or expediting urgent tests, which can involve actually taking patients to those tests.

The nurse coordinator also gives advice on results to junior doctors, talks to patients and families about results, prescribes urgent drugs (where no relevant doctor is available) and liaises with GPs and other units as required.

\section{Nurse-led telephone clinics and 'hot' clinic slots}

Patients who do not require inpatient care are considered for early discharge with nurse follow-up by telephone, at nurse-led acute or so-called 'hot' outpatient clinic slots with consultant supervision or in a consultant-delivered clinic (templates changed to accommodate 'hot' slots).

\section{The role of epilepsy nurses in acute care}

Every patient admitted to either the AMU or RAMU following a fit with a prior diagnosis of epilepsy or new-onset epilepsy (but not single seizures) are seen, usually on the same day, by an acute epilepsy nurse. The epilepsy nurses give acute advice and arrange early follow-up both by telephone and in nurseled clinics. Study data were collected for the periods March to August in 2015 and 2016, the equivalent 6-month periods before and after the nurses started.

\section{The acute neurophysiology service}

The consultant of the day is able to identify patients who would benefit from an EEG or EMG in order to facilitate diagnosis or management, including early discharge. The neurophysiology lead (unit manager and lead clinical physiologist) is contacted by the consultant or the nurse coordinator. Tests are usually carried out on the day of referral or the subsequent one. The unit is located close to the AMU often making it possible to bring patients for tests without delays involved in waiting for porters.

\section{Key data collected and outcome objectives}

Data had been collected for 2014, the year before the nurse coordinator started. We have compared these data to 2015 as well as 6-month figures for January to June 2016. This encompasses the first 18 months of having a neurology nurse coordinator in post. Data collected includes:

$>$ total monthly neurology referrals

$>$ referrals from the AMU compared with general wards

$>$ the delay between a patient being referred and being seen by the consultant

$>$ the use of telephone and acute 'hot' clinic slots

$>$ re-admission rates

> length of stay from March to August for 2014 and 2015.

For the subset of patients with epilepsy, data on length of stay and readmission rates were collected from March to August for 2015 and 2016.

Ethical approval was not necessary for this study as it was a quality improvement project.

\section{Results}

\section{Referral numbers}

For 2014, there was an average of 60 referrals (range 51-79) per month; the total for the year was 724. In 2015, the year after the nurse started, 77 patients were seen per month (range 63-95)

- annual total 924 (Fig 1A). This was a 22\% increase in referral numbers, an average increase from three patients per day to four patients. The number of referrals continued to rise for the first 6 months of 2016. A total of 514 referrals were made, which is an average of 86 per month and a further increase of $11 \%$.

In 2014, the vast majority of patients being referred had already been moved to a general medical ward from the AMU; only 169 patients $(23 \%)$ were seen while still on the AMU. The percentage seen on the AMU has risen steadily: in 2015 it was 356 patients (39\%) and between January and June 2016 it was 230 patients $(45 \%-230 / 514$; Fig $1 \mathrm{~B})$.

\section{Delay between referral and patient being seen}

The delay between referral and a patient being seen is expressed as the percentage of patients seen on the day of referral (day 0 ). As this is a 5-day service, a patient referred on Friday and seen on Monday appears as a delay of 1 day. There was a dramatic increase in patients seen on the day of referral: in 2014, 59\% of patients were seen on the day of referral, which rose to $71 \%$ by 2015 . This figure continued to rise in the first 6 months of 2016: 389 of 514 patients were seen on the same day $(76 \%)$. The percentage waiting 48 hours or more dropped from $11 \%$ to less than $1 \%$ (Fig 1C).

\section{Time during the day when patients' receive consultant} review

In 2015, the time in the day when patients were seen by the consultant with the nurse coordinator was between $10 \mathrm{am}$ and 
Fig 1. Referrals to the hyperacute neurology team 2014-16. A - mean monthly referrals; $B$ - percentage of patients seen on the acute medical unit; $\mathrm{C}$ - percentage of patients seen on day of referral.

\section{A}

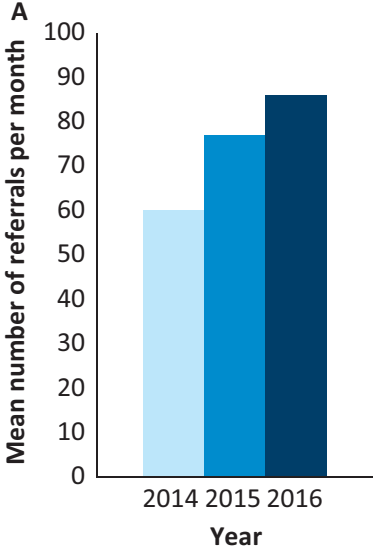

B

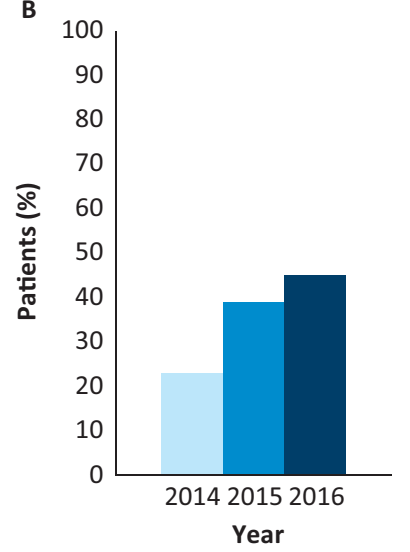

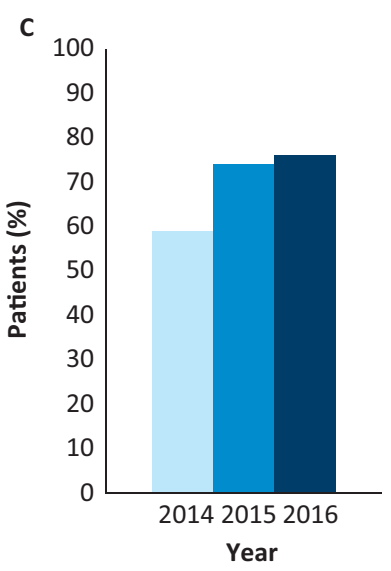

$12 \mathrm{pm}(16 \%), 12$ and $2 \mathrm{pm}(57 \%), 2$ and 4 pm (21\%) and after 4 pm $(6 \%)$.

\section{Underlying principal diagnoses}

Principal diagnoses are shown in Fig 2, for both 2014 and 2015. Numbers are based on the first 250 referrals made from August to November (in 2014 and in 2015). Epilepsy was the most common diagnosis (30\% of admissions) followed by headache (9\%).

\section{Nurse-led telephone and 'hot' clinic slot numbers}

The number of patients who required urgent follow-up by the nurse coordinator in 2015 was 114 . These were either contacted in telephone clinics or were seen in 'hot' clinic slots within 2 weeks of discharge from the AMU; this was an average of 11 patients per month. In total, 37 patients were seen in person and 77 by telephone. This resulted in the clinic templates being amended to change four routine slots to 'hot' slots. The principal symptoms for the use of these 'hot' slots were seizures and headaches.

\section{Length of stay and readmission rate}

The average length of stay for patients referred to the acute neurology service from March to August in 2014 and 2015 was 17 days in both years with no significant change despite the enhanced service. Readmission rates within 30 days were also unchanged (20\%). Post-hoc analysis of the $50 \%$ of the cohort who had the shorter length of stay revealed a significant reduction in length of stay from 3.6 to 3.1 days $(p=0.04)$ in this subgroup.

\section{Epilepsy nurse outcomes}

The epilepsy data presented is only for the 6-month period March to August 2016 compared with the same 6-month period in 2015 and as such must be viewed as preliminary. A total of 86 patients who were admitted to the AMU in 2016 were seen
Fig 2. Diagnosis for patients seen by the hyperacute neurology team.

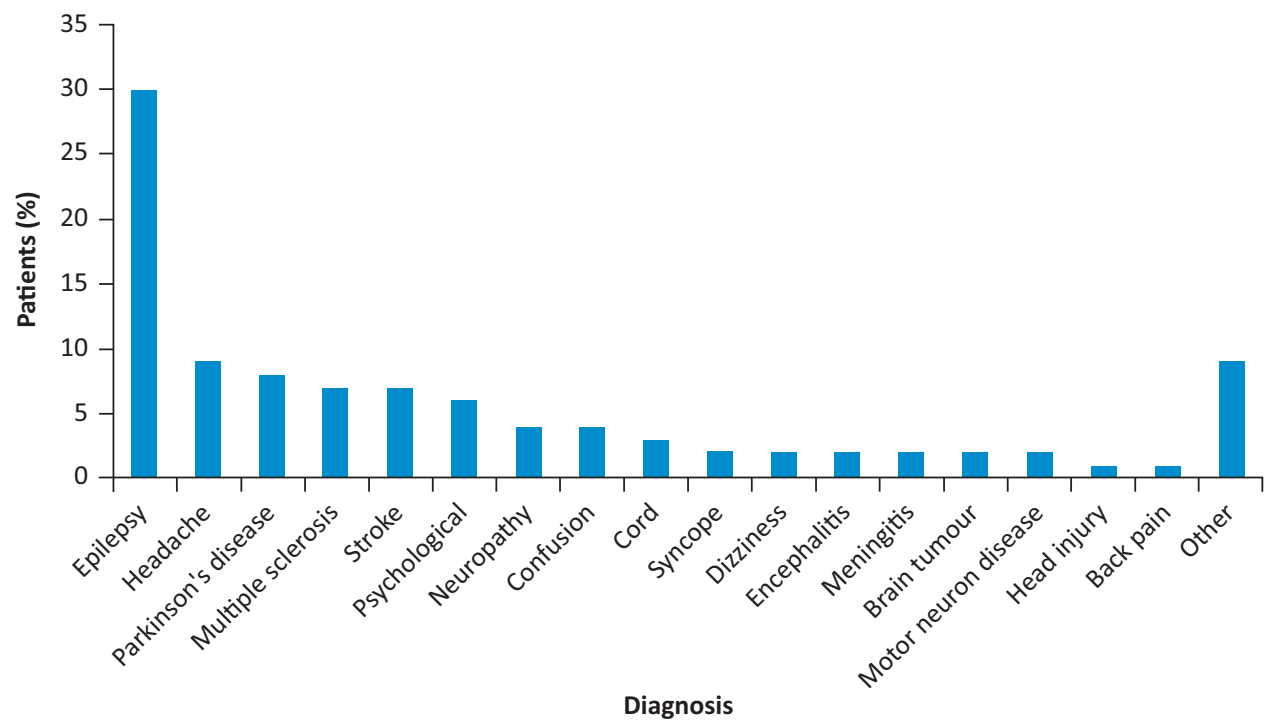




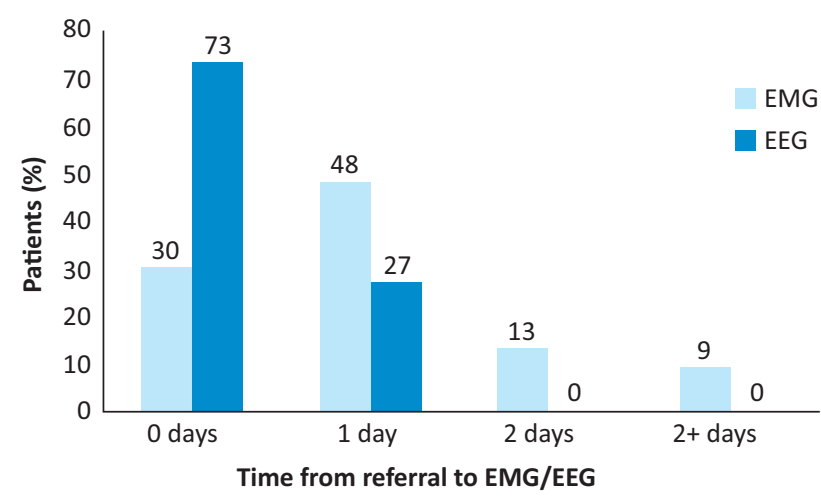

Fig 3. Time from neurophysiology referral to electroencephalogram (EEG) and electromyogram (EMG).

by the acute epilepsy nurses; 80 of these patients were seen on the day of referral (93\%). These 86 patients represent about $17 \%$ of all the neurology referrals for those 6 months suggesting incomplete pick up of patients with epilepsy in this the first 6 months. The most important change was the reduction in total bed days occupied by patients with a primary diagnosis of epilepsy by $32 \%$ from 632 in 2015 to 427 in 2016. The total number of attendances to the emergency department was reduced by $12 \%$ (from 107 to 94 ) and the number of admissions was reduced by $13 \%$ (from 143 to 124). Average length of stay was reduced from 4.4 days to 3.5 days $(20 \%)$.

\section{Acute neurophysiology service}

Between 1 January and 31 October 2016, 51 EEGs were requested by the neurology consultant on call for the HANT for a particular day. Of these, $73 \%$ were carried out on the day of referral and $27 \%$ the following day. Of the 23 EMGs requested, $30 \%$ were done on the day of referral, $48 \%$ the subsequent day and $22 \%$ patients waited longer (Fig 3).

\section{Financial cost of establishing the HANT}

The money invested in setting up the HANT is outlined in Table 1.

\section{Discussion}

This study represents a 'proof of concept' demonstrating, firstly, that it is possible to set up a HANT in a typical district general hospital and run it successfully for 18 months. In this pilot, we have achieved this without major changes to consultant job plans, but if this model is to be widely accepted, a shift towards more programmed activities for acute neurological care will probably be necessary. Secondly, we have shown that a HANT can achieve major improvements in key indicators of care.

The improvements in performance demonstrated so far are numerous. The percentage of patients referred from the acute service has risen from $22 \%$ to $45 \%$, greatly facilitating chances of early intervention and reducing length of stay. We anticipate these numbers will continue to rise because, despite the number of referrals increasing, we are still only reviewing a minority of the total number of patients with neurological diagnoses

Table 1. Outline of the money invested in setting up

the hyperacute neurology team

Post

One WTE band 8 nurse - neurology nurse coordinator

Two WTE band 7 - community epilepsy nurses ( $30 \%$ of time on acute work)

Two consultant neurology sessions to train and clinically supervise the nurses

Administrative support for the acute work of three nurses

Total

CCG = clinical commissioning group; $\mathrm{WTE}=$ whole-time equivalent

in hospital. The number of patients seen on the day they are referred has gone up from $59 \%$ to $76 \%$. Of these, $73 \%$ were seen before $2 \mathrm{pm}$. This is vital so that management decisions can be implemented and relevant diagnostic tests carried out on the same day as the consultant sees the patient.

The preliminary improvements shown in epilepsy care are encouraging, with a reduction in the total length of stay of $32 \%$ for patients admitted with a primary diagnosis of epilepsy. Other studies, however, have not consistently shown such changes. ${ }^{5}$

It is disappointing that total length of stay did not change. We suspect in the longer term the length of stay would go down for short-stay patients but may not change for longer-stay patients with multiple pathologies and potential social issues.

This HANT brings together six relatively novel approaches to acute neurological care:

\section{The senior acute neurology nurse coordinator role}

This member of the team is pivotal. The nurse coordinator is the key link between all the AMU staff and the neurology team. They triage the patients and are able to book tests and prescribe drugs. We believe the use of a senior nurse coordinator has major advantages over other potential alternatives, which include neurology trainees, clinical associates and non-consultant grade doctors. The critical key to the HANT's success lies in its ability to streamline the often complex care pathways involved in patients getting the most appropriate care. This involves a combination of clinical skills but also administrative components, such as liaising with bed managers, nursing staff, pharmacy managers and even on occasion commissioners. This dual skill set possessed by a senior nurse with both clinical and administrative experience has proved a key learning point for our service. An experienced physician associate may also be able to fill such a role.

\section{The acute epilepsy nurses}

Because it is increasingly clear that seizures are the largest cause of acute neurological admissions (about 30\% from our data), the establishment of a designated acute epilepsy nurse-run service to target the needs of these patients seems obvious. Our data suggest a major reduction in key outcomes is possible.

\section{Acute neurophysiology service}

In most places, neurophysiology departments currently play a very limited role in acute neurological service 
provision; services are mainly provided to outpatient and established admissions. Our study has shown that with neurology consultants alone making urgent requests, the extra workload is relatively small and manageable (5.1 extra EEGs and 2.3 extra EMGs per month). The service was able to carry out $85 \%$ of both EEGs and EMGs within 72 hours of admission. These tests probably do not represent extra new work but rather a shift towards earlier appointments for patients, many of whom would have been seen at a later date.

4 A fully consultant delivered acute neurology service Although the service is $100 \%$ consultant delivered, the ability to provide the rapid service relies heavily on the nurse coordinator's ability to triage patients before they are seen and activate management plans after they have been seen.

5 Service being physically based in or very close to the AMU The success of the service is largely based on all of the AMU and RAMU staff knowing that the nurse coordinator is always around and being able to refer patients as and when they feel it is indicated. This approachable system is more enabling than the more formal referral systems.

6 Telephone and 'hot' slots

In order to achieve the early discharge of patients, there needs to be an expansion of both nurse- and consultantled telephone and 'hot' slots. In this context, a hot slot is re-labelling a slot in the usual consultant's clinic template enabling patients to be booked within 3 days. This is a similar concept to the long established rapid access neurology clinics run at Addenbrooke's Hospital, Cambridge, UK. ${ }^{6}$ Depending on the number of consultants in the trust, this may affect referral-to-treatment time but if consultant numbers are nearing the Association of British Neurologists numbers of 1 per 70,000 (a target proposed in their paper Local adult neurology services for the next decade, 2011) then like our experience, referral-to-treatment time is not affected adversely. The total numbers of 'hot' patients seen in 2015 by the nurse coordinator and the consultants was relatively small - 11 per month, which is a relatively manageable number. These figures do not include the extra work done for patients with epilepsy.

We are keen to look at further developments - how to improve cover arrangements for the nurse coordinator (currently provided on an ad hoc basis by one of the epilepsy nurses) and how we could move to 7-day working. Our favoured model would be for two adjoining district general hospitals to share an on-call weekend rota. A pool of 8-10 consultants is probably a realistic number while our current number of four seems unrealistic. The practical and financial details of how to provide cover to two sites would need to be considered.

We can find no similar studies but feel this model is worthy of widespread discussion. There is no competition between these two models of acute neurological care - HANTs and HANUs. HANTs offer a potential easy 'quick fix' with relatively low cost implications and may be well suited to district general hospitals, while HANUs may be a more realistic option for tertiary centres. In the long term, it is obviously ideal for all patients with acute neurological problems to be looked after by teams with specialist neurological expertise.

There are four prerequisites for setting up a successful HANT:

1 A major commitment from all of the consultants in the hospital to acute work, currently Monday to Friday, in addition to other commitments.
2 A change in the working patterns of designated specialist neurology nurses from routine outpatients to acute admission work, which requires significant extra training and supervision.

3 A real desire by the acute physicians, the neurophysiology services and the neuroimaging services to be part of an integrated acute neurological care pathway.

4 The increasing use by both nurses and consultants of telephone clinics and 'hot' slots to accommodate patients discharged early from AMU.

\section{Conclusions}

This study has shown how a senior acute neurology nurse coordinator, acute epilepsy nurses and changing the neurology consultants' working pattern together with an acute neurophysiology service and engaged acute physicians has helped improve the care of neurological patients, especially those with epilepsy.

\section{Conflicts of interest}

The authors have no conflicts of interest to declare

\section{Author contributions}

$\mathrm{AB}, \mathrm{AT}, \mathrm{CS}$ and MS collated the data and AN analysed the data. AB, $A N$ and FS wrote the initial draft of the paper. All authors (AB, AN, AT, BKM, CS, MS, FS) have made substantial contributions, revised the paper, contributed to the interpretation of the data and agree to all aspects of the work.

\section{Acknowledgements}

We wish to thank Croydon Clinical Commissioning Group, Dr Reza Motazed (lead consultant in acute medicine) and Dr Francesca Mastrolilli (consultant neurologist) for their unfailing support during the period of this study and beyond

\section{References}

1 The Neurological Alliance. Neuro numbers. London: The Neurological Alliance, 2014. www.neural.org.uk/store/assets/files/381/original/ Final_-_Neuro_Numbers_30_April_2014_.pdf [Accessed 12 May 2017].

2 Royal College of Physicians. Local adult neurology services for the next decade. Report of a working party. London: RCP, 2011.

3 Reveruzzi B, Pilling S, Davie C, Sud P. Hyper acute neurology unit (HANU) evaluation. London: UCL Partners, 2016. www.londonscn.nhs. uk/wp-content/uploads/2016/10/HANU-Evaluation-Final-Report.pdf [Accessed 12 May 2017].

4 Gregory R, Nicholl D, Lawrence J. Acute Neurology services survey 2017. London: Association of British Neurologists, 2017.

5 Noble A, McCrone P, Seed PT, Goldstein LH, Ridsdale L. Clinical and cost-effectiveness of a nurse led self-management intervention to reduce emergency visits by people with epilepsy. PLoS ONE 2014;9:e90789.

6 Axinte LT, Fiddes BD, Donaghy A et al. Experience from two decades of the Cambridge Rapid Access Neurology Clinic. Clin Med 2015;15:43741.

Address for correspondence: Dr Arani Nitkunan, Neurology Department, Croydon University Hospital, Mayday Road, Croydon CR7 7YE, UK.

Email: anitkunan@nhs.net 